\title{
Impact of Cardiopulmonary Resuscitation Duration on the Neurological Outcomes of Out-of-Hospital Cardiac Arrest
}

HISSAH ALBINALI ( $\square$ HISSAH.ALBINALI@HOTMAIL.COM )

Royal Commission Hospital in Jubail

Arwa Alumran

Imam Abdulrahman Bin Faisal University

Saja AlRayes

Imam Abdulrahman Bin Faisal University

Original Research

Keywords: Cardiac arrest, Patient Outcomes, Cardiopulmonary resuscitation, Neurological outcome, Cerebral Performance Category

Posted Date: June 8th, 2021

DOI: https://doi.org/10.21203/rs.3.rs-566168/v1

License: (c) (i) This work is licensed under a Creative Commons Attribution 4.0 International License. Read Full License

Version of Record: A version of this preprint was published at International Journal of Emergency Medicine on March 19th, 2022. See the published version at https://doi.org/10.1186/s12245-022-004184. 


\section{Abstract}

Background: Patients experiencing cardiac arrest outside medical facilities are at greater risk of death and might have negative neurological outcomes. Cardiopulmonary resuscitation duration affects neurological outcomes of such patients, which suggests that duration of CPR may be vital to patient outcomes.

Objectives: The study aims to evaluate the impact of cardiopulmonary resuscitation duration on neurological outcome of patients who have suffered out-of-hospital cardiac arrest.

Methods: Data were collected from emergency cases handled by a secondary hospital in industrial Jubail, Saudi Arabia, between 2015 and 2020. There were 257 out-of-hospital cardiac arrest cases, 236 of which resulted in death.

Results: Bivariate analysis showed no significant association between cerebral performance category (CPC) outcomes and duration of CPR, gender and cause of death whereas there is statistically significant between CPC and age. ( $p=0.001)$. However, a good CPC outcome was reported with a (mean) limited duration of 8.1 min of CPR; whereas, poor CPC outcomes were associated with prolonged periods of CPR, 13.2 min (mean). Similarly, youthfulness was associated with good CPC outcomes as revealed by the mean age of 5.8 years, whereas a mean rank of 14.9 years was aligned with a poor CPC outcome.

Conclusion: Cardiopulmonary Resuscitation Duration out-of-hospital cardiac arrest does not significantly influence the patient neurological outcome in the current study hospital. Other variables may have a more significant effect.

\section{Introduction}

Out-of-hospital cardiac arrest (OHCA) is a major public health concern across the world. Statistics demonstrate that an average of 330,000 people in the United States and 275,000 people in Europe experience OHCAs every year (Kashiura et al., 2017). Moreover, the same statistics demonstrate that the survival rates for OHCAs are low. Cardiac arrests occur when the heart has stopped pumping blood to the body, accounting for many deaths across the world; most cardiac arrests happen outside hospital facilities (Hayashi et al., 2020). According to Paratz et al. (2020), cardiac arrests account for $20 \%$ of all Western deaths. Cardiac arrest is the result of a number of factors, and several scholars have attempted to determine the relationship between various prehospital factors such as the first recorded rhythm, age, and the survival rate after cardiac arrests. OHCAs affect society negatively and in a significant way (Shida et al., 2019). For instance, patients' family members experience emotional burdens and the medical staff suffers vital implications. To rescue a cardiac arrest patient, medical professionals perform $\mathrm{CPR}$, which includes rescue breathing such as mouth-to-mouth resuscitation and chest compressions (Zhan et al., 2017). 
Patients experiencing cardiac arrest might have negative neurological outcomes. The outcomes of cardiac arrest are mainly related to its effects on the neurological system. The neurological outcome can be good or poor, depending on a variety of factors. Therefore, it is important to perform this assessment in situations where CPR procedures have been administered. The cerebral performance category (CPC) scale is tool for evaluation of neurological damage after cardiac arrest and describes patients mental ability from $\mathrm{CPC}=1$ to $\mathrm{CPC}=5$.

A study by Akin et al. (2021) conducted on 25 patients indicated that $41 \%$ had good neurological outcomes with a Cerebral Performance Category score of less than or equal to 2 . The good outcomes were related to high-quality care and recognition of the cardiac arrest within the shortest time possible.

Park et al. (2018) found that of 65 cardiac arrest patients investigated, only $24.6 \%$ had positive neurological outcomes, whereas $75.4 \%$ had poor outcomes. Most poor prognoses are related to the withdrawal of life-sustaining therapy after the cardiac arrest. Therefore, it is important to carry out a neurological examination after cardiac arrest, including an examination of the different reflexes and motor responses. This examination can prompt interventions that are important to prevent further complications to the patient.

Duration plays a significant and crucial role in the identification of neurological outcomes after cardiac arrest. Health care workers should record good or poor neurological outcomes after a patient's cardiac arrest. A study by Yukawa et al. (2017) indicated that patients had a good neurological outcome when the CPR duration was between 40 min. It also is important to initiate CPR within 40 min of the onset of cardiac arrest, either prehospital or in the hospital. With a shorter duration of CPR, the outcomes are positive.

Neurological outcomes manifest differently among different age groups. Ichord et al. (2018) found that pediatric patients who had experienced cardiac arrest showed mild impairments in their neurological outcomes. Therefore, the neurological results can be positive in pediatric patients. Hirlekar et al. (2017) found that neurological outcomes among the elderly progress with age. They examined people aged 70 years and over who had suffered a cardiac arrest. The results indicated that of those aged 70-79 years, $80-89$ years, and 90 years, $92 \%, 93 \%$, and $88 \%$, respectively, had good neurological outcomes. These results proved that the elderly can have good neurological outcomes.

Males and females experience different manifestations of cardiac arrest. Lei et al. (2020) carried out a study to determine gender differences in neurological outcomes and found that females are more likely to have favorable neurological outcomes than males. After an admission, the females received fewer interventions such as coronary artery angiography and percutaneous coronary intervention, which are important for cardiac arrest patients.

In cases of trauma, the outcomes can be a bit different. Brain injury is one of the traumas that can cause cardiac arrest. According to Zhao et al. (2021), among 42 patients who had a traumatic cardiac arrest, 
eight survived until the time of discharge, and only seven survived with good neurological outcomes. Therefore, it is important to resuscitate those with traumatic injuries because some have good outcomes.

Poor outcomes result from a lack of proper intervention and early detection of cardiac arrest. Death results from too much trauma or the presence of medical conditions that complicate intervention (Oh et al., 2017). Pediatric patients can be linked to poor outcomes because of their ongoing neurodevelopment, which cardiac arrest can greatly affect. It causes most of their Cerebral Performance Category scores to be $3-4$.

The above mentioned literature provides evidence of the importance of understanding the overall effects of CPR duration on neurological outcomes. Health care professionals have made attempts to terminate CPR's use to stop the effects that can arise from its administration. Nonetheless, various organizations have argued that the procedure should not be discontinued entirely; instead, health care professionals should be granted the right to weigh the benefits of its administration as part of the CPR protocol (Kashiura et al., 2017). No study has been conducted in Industrial Jubail, Saudi Arabia, to determine the impact of CPR duration on neurological outcomes of OHCA and the influencing factors. This study sought to explore the relationship between CPR duration and the achievement of neurologically favorable outcomes at a secondary hospital in Industrial Jubail.

\section{Research Methodology \\ Research Design}

This is a quantitative cross-sectional design to evaluate the impact of CPR duration on neurological outcomes of Out of Hospital Cardiac Arrest (OHCA).

\section{Study Setting}

This research was carried out at a major hospital in Industrial Jubail, Eastern Saudi Arabia. The study institution has eight ambulances and offers care for various groups of patients who are critically ill and require comprehensive stabilization. About two to three prehospital cardiac arrest incidents occur each month; the attending Emergency Medical Service (EMS) specialist will request assistance and initiate efforts aimed at resuscitating the patient in collaboration with other members of the team. According to AHA guidelines, when providing CPR, the EMS specialist in charge is required to fill out a CPR form and ensure that it is duly completed after the event.

\section{Study Participants}

This study included only the patients' OHCA data, which was extracted from a hospital database. Both pediatric and adult patients of both genders were included. 


\section{Variables}

The dependent variables assessed in this study included patients' neurological outcomes, which were measured using the Cerebral Performance Category (CPC) scale (Edgren et al., 1994). The outcome variable was categorized according to either good or poor neurological outcomes based on previous studies (Xue et al. 2013).

The independent variables assessed in this study included patients' gender, age, and the duration of CPR measured in minutes until the return of spontaneous circulation was achieved in prehospital settings or after hospital admission.

\section{Data Collection}

The hospital's electronic medical database was utilized retrospectively to collect the participating patients' data; this was important because it provided easy access to important demographic data and patient outcome information. The cardiac arrest cases sampled in this study were reported between 2015 and 2020 .

\section{Instruments}

The cerebral performance category (CPC) scale was used to assess neurological outcomes from resuscitation attempts at hospital discharge. According to numerous studies (Edgren et al., 1994), a CPC score of 1 or 2 shows a good neurological outcome (favorable outcome), whereas a score of 3, 4, or 5 shows a poor neurological outcome (unfavorable outcome, often severe neurological outcome, or death).

\section{Procedure and Timeline}

The cerebral performance category (CPC) assessment of patients' neurological outcomes was done at hospital discharge. Data were gathered during a 2-month period between January and February 2021.

\section{Ethics and Limitations}

Ethical approval was obtained from the Institutional Review Board at Imam Abdulrahman Bin Faisal University, Dammam, Saudi Arabia (IRB-PGS-2021-03-048) and the Institutional Review Board at Royal Commission Hospital, Industrial Jubail, Saudi Arabia (IRB-RCH-013). The medical chart research was carried out confidentially without the exposure of any of the participants' personal information.

\section{Analysis}


To determine and illustrate a descriptive summary of the findings, means and standard deviations were calculated for continuous variables, Counts and frequencies were used for categorical variables. Additionally, bivariate analysis was carried out to evaluate the association between the cerebral performance category (CPC) results indicating neurological outcomes and patient's ender using $\chi^{2}$ test, and between the effect of CPR duration and the patients age on the neurological outcome were assessed using Mann-Whitney U test. IBM SPSS Statistics (Version 25) (IBM Corp, 2017) was utilized to carry out the analysis.

\section{Results}

Of the 257 patients included in this study, $184(71.6 \%)$ were males and $73(28.4 \%)$ were females. The mean and standard deviation of the patients' ages were $48.4 \pm 23.2$ years (age range: $7-94$ years). The overall mean and standard deviation of the duration of CPR was found to be $29.3 \pm 9$ min (with an actual range of 9-60 min). Almost half of the patients in the study had an OHCA because of a medical condition ( $n=144,56 \%)$, while the rest was caused by Trauma $(n=113,44 \%)$. The patients' characteristics are summarized in Table 1.

Table 1

\begin{tabular}{|l|l|}
\hline \multicolumn{1}{l}{-Demographic Characteristics of Study Participants } \\
\hline $\begin{array}{l}\text { Vender } \\
\text { Male }\end{array}$ & $\begin{array}{l}\text { Frequency (\%) } \\
\text { Female }\end{array}$ \\
\hline $\begin{array}{l}\text { Cause of OHCA } \\
\text { Medical }\end{array}$ & $73(28)$ \\
Trauma & $144(56)$ \\
\hline Age (years) & $113(44)$ \\
\hline Duration of CPR (minutes) & $29.3 \pm 9$ (range: 9-60) \\
\hline
\end{tabular}


Table 2

- Association Between Demographic Factors, Cause of Death, and Duration of CPR With CPC

\begin{tabular}{|c|c|c|c|}
\hline & \multicolumn{2}{|c|}{$\mathrm{CPC}^{*}$ Outcome } & \multirow[t]{2}{*}{$\chi^{2}$ (P-value) } \\
\hline & Good & Poor & \\
\hline & n (\%) & $\mathrm{n}(\%)$ & \\
\hline Gender & 0 (500) & $2(5001)$ & $.175(.676)$ \\
\hline Female & ) & (0) & \\
\hline Male & $6(40 \%)$ & $9(60 \%)$ & \\
\hline Cause of death & $2(22.2 \%)$ & $7(77.8 \%)$ & $2.738(0.098)$ \\
\hline Medical & $7(58.3 \%)$ & $5(41.7 \%)$ & \\
\hline \multicolumn{4}{|l|}{ Trauma } \\
\hline & Mean Rank & Mean Rank & Mann-Whitney U test (P-Value) \\
\hline Duration of CPR (minutes) & 8.1 & 13.2 & $27.500(0.059)$ \\
\hline Age (years) & 5.8 & 14.9 & $7.000(0.001)$ \\
\hline
\end{tabular}

Half of the female survivors had good cerebral performance category (CPC) outcome ( $n=2,50 \%$ ), while only $41 \%$ of males survivors $(n=7)$ had good outcome. Nevertheless, there was no statistically significant difference in cerebral performance category (CPC) outcomes between gender groups $(p=0.748)$.

In addition, the assessment was done on the cause of the OHCA, i.e., either medical or traumatic causes. The cerebral performance category (CPC) outcomes in patients who developed an OHCA due to medical causes showed that two (22.2\%) individuals had good CPC outcomes and seven (77.8\%) recorded poor CPC outcomes. The CPC outcomes were a bit better in patients who developed OHCA due to traumatic causes, more than half had good CPC outcomes $(n=7,58.3 \%)$. However, there was no statistically significant difference in CPC outcomes between the two causes of OHCA (medical or trauma; $p=0.098$ ).

Regarding the duration of CPR in minutes, a good CPC outcome was reported with a (mean) limited duration of 8.1 min of CPR; whereas, poor CPC outcomes were associated with prolonged periods of CPR, 13.2 min (mean). Similarly, youthfulness was associated with good CPC outcomes as revealed by the mean age of 5.8 years, whereas a mean rank of 14.9 years was aligned with a poor CPC outcome; this difference between age groups was statistically significant $(p=0.001)$.

\section{Discussion}


Because cardiac arrest continues to cause a significant number of deaths in Saudi Arabia, it is important to investigate the effectiveness of CPR procedures in saving the lives of affected individuals. To achieve that goal, this study on the effects of CPR duration on neurological outcomes, together with the existing literature, offers insights concerning the termination of the medical procedure. This study aimed to investigate the impact of CPR duration on neurological outcomes to demonstrate those outcomes in cardiac arrest patients. This was achieved through the utilization of a qualitative cross-sectional design to assess the impact of CPR duration on neurological outcomes.

The dependent variables in the study included good or poor neurological outcomes that were assessed against independent variables such as age, sex, and duration of CPR.

This study inferred that shorter CPR durations led to favorable neurological outcomes and survival and study reported a strong association between $\mathrm{CPC}$ scores and rapid recovery trends. A good outcome was reported with a limited duration of 8.1 min of CPR administration, whereas poor CPC outcomes were associated with prolonged periods of CPR with an average of $13.2 \mathrm{~min}$. These results correspond with those from a study by Xue et al. (2013), who established that shorter CPR durations result in higher chances of survival compared to increased CPR durations. This is also in agreement with a study by Scott et al. (2015), who established that individuals who were exposed to shorter CPR durations had higher chances of recovering from cardiac arrest than those exposed to prolonged CPR. Moreover, CPC outcomes in the patients who succumbed to medical issues revealed that $22 \%$ of the sample had good CPC outcomes and 78\% recorded poor CPC outcomes. In harmony with the findings of Scott et al., good CPC outcomes resulted in faster recovery among cardiac arrest patients.

Findings from the research also demonstrated the relationship between the independent variables in this study and CPR outcomes. An influential factor in the recovery trend, age differences among subjects were translated in the results. According to the findings, youthfulness is associated with good CPC outcomes and a mean rank of 5.8 years, whereas a mean rank of 14.9 years was aligned with poor CPC outcomes among test subjects. This study also concluded that other underlying medical conditions limited recovery trends in older patients. This was in line with the findings of Goto et al. (2018). According to Cheema et al. (2019), patients with numerous comorbidities have lower chances of recovering after CPR administration. In addition to age and underlying medical conditions, this study demonstrated a significant difference in recovery rates between male and female patients. Outcomes in patients who survived after CPR administration showed that $50 \%$ of the women who recovered had good CPC outcomes. However, $58.8 \%$ of the men with poor CPC outcomes survived, and only $41.2 \%$ of the men with good CPC outcomes survived. These results align with the findings of Morrison et al. (2016), who reported significant differences in recovery trends between male and female patients. This study has also demonstrated such significant differences.

\section{Conclusion}


This study indicates that shorter CPR durations lead to favorable neurological outcomes and survival among cardiac arrest patients. Survival among such patients is significantly affected by preexisting medical conditions (Matos et al., 2013). However, favorable neurological outcomes and recovery rates were observed to be significantly influenced by intervention strategies adopted by clinicians. This study, therefore, recommends the adoption of intervention strategies that not only help cardiac arrest patients overcome underlying medical conditions but also bring about favorable neurological outcomes and survival. Moreover, this study concluded that patients' demographic data, such as gender and age, significantly influence outcomes in cardiac arrest patients. This study recommends the adoption of differentiated intervention strategies for cardiac arrest patients to enhance clinicians' capacities to address specific medical needs that may influence neurological outcomes in individual patients.

This study also suggests that the effects of extended CPR administration are exacerbated in elderly patients. Older people are predisposed to multiple health care needs that increase their vulnerability to cardiac arrest (Al-Mulhim et al., 2019). Longer CPR durations among such patients lower their chances of survival from cardiac arrest. Because CPR largely contributes to OHCA treatment and the return of spontaneous circulation, this study recommends a keen analysis of CPR duration by medical practitioners to avoid complications, particularly in elderly patients. Moreover, health organizations ought to teach CPR administration and the average beneficial duration to members of the public to inform them of the best out-of-hospital intervention strategies for individuals suffering from cardiac arrest.

There is a need for further investigation into other factors. First, multifactorial traits such as underlying health conditions were not considered in this research. It was assumed that OHCA patients had received advanced life support according to CPR guidelines. Second, the possibility of uncontrolled confounders cannot be ruled out because of the nature of the participants. For instance, data on various factors such as the location where the OHCA occurred and preexisting comorbidities as well as the quality of the first CPR administered were not considered in this study. It is evident that the quality of the first CPR administered has a substantial impact on the patient's recovery.

\section{Abbreviations}

$\mathrm{CPC}=$ Cerebral Performance Category

\section{Declarations}

\section{Disclosure/conflict of interest:}

the authors declare that there is no conflict of interest relevant to this work.

\section{Acknowledgement:}

the authors would like to thank www.papercheck.com for their proof reading services 


\section{References}

1. Akin, M., Garcheva, V., Sieweke, J. T., Adel, J., Flierl, U., Bauersachs, J., \& Schäfer, A. (2021). Neuromarkers and neurological outcome in out-of-hospital cardiac arrest patients treated with therapeutic hypothermia-Experience from the HAnnover COoling REgistry (HACORE). Plos One, 16(1), Article e0245210. https://journals.plos.org/plosone/article?id=10.1371/journal.pone.0245210

2. Cheema, M. A., Ullah, W., Abdullah, H. M. A., Haq, S., Ahmad, A., \& Balaratna, A. (2019). Duration of inhospital cardiopulmonary resuscitation and its effect on survival. Indian Heart Journal, 71(4), 314319.

3. Al-Mulhim, M. A., Alshahrani, M. S., Asonto, L. P., Abdulhady, A., Almutairi, T. M., Hajji, M., \& Al-Qahtani, L. B. (2019). Impact of epinephrine administration frequency in out-of-hospital cardiac arrest patients: a retrospective analysis in a tertiary hospital setting. Journal of International Medical Research, 47(9), 4272-4283.

4. Edgren, E., Hedstrand, U., Kelsey, S., Sutton-Tyrrell, K., Safar, P., \& BRCTI Study Group. (1994). Assessment of neurological prognosis in comatose survivors of cardiac arrest. The Lancet, 343(8905), 1055-1059. https://doi.org/10.1016/s0140-6736(94)90179-1

5. Goto, T., Morita, S., Kitamura, T., Natsukawa, T., Sawano, H., Hayashi, Y., \& Kai, T. (2018). Impact of extracorporeal cardiopulmonary resuscitation on outcomes of elderly patients who had out-ofhospital cardiac arrests: A single-centre retrospective analysis. BMJ Open, 8(5).

6. Hirlekar, G., Karlsson, T., Aune, S., Ravn-Fischer, A., Albertsson, P., Herlitz, J., \& Libungan, B. (2017). Survival and neurological outcome in the elderly after in-hospital cardiac arrest. Resuscitation, 118, 101-106. https://doi.org/10.1016/j.resuscitation.2017.07.013

7. Ichord, R., Silverstein, F. S., Slomine, B. S., Telford, R., Christensen, J., Holubkov, R., Dean, J. M., \& Moler, F. W. (2018). Neurologic outcomes in pediatric cardiac arrest survivors enrolled in the THAPCA trials. Neurology, 91(2), e123-e131. https://doi.org/10.1212/WNL.0000000000005773

8. Kashiura, M., Hamabe, Y., Akashi, A., Sakurai, A., Tahara, Y., Yonemoto, N., ... Morimura, N. (2017). Association between cardiopulmonary resuscitation duration and one-month neurological outcomes for out-of-hospital cardiac arrest: A prospective cohort study. BMC Anesthesiology, 17(1), 1-8.

9. Lei, H., Jiahui, H., \& Danyan Xu. (2020). Sex differences in survival after out-of-hospital cardiac arrest: a meta-analysis. Critical Care. https://ccforum.biomedcentral.com/articles/10.1186/s13054-02003331-5

10. Matos, R. I., Watson, R. S., Nadkarni, V. M., Huang, H. H., Berg, R. A., Meaney, P. A., \& Spinella, P. C. (2013). Duration of cardiopulmonary resuscitation and illness category impact survival and neurologic outcomes for in-hospital pediatric cardiac arrests. Circulation, 127(4), 442-451.

11. Morrison, L. J., Schmicker, R. H., Weisfeldt, M. L., Bigham, B. L., Berg, R. A., Topjian, A. A., ... Resuscitation Outcomes Consortium Investigators. (2016). Effect of gender on outcome of out of hospital cardiac arrest in the Resuscitation Outcomes Consortium. Resuscitation, 100, 76-81. 
12. Oh, S. H., Park, K. N., Lim, J., Choi, S. P., Oh, J. S., Cho, I. S., ... Kim, S. H. (2017). The impact of sex and age on neurological outcomes in out-of-hospital cardiac arrest patients with targeted temperature management. Critical Care, 21(1). https://doi.org/10.1186/s13054-017-1860-5

13. Paratz, E. D., Rowsell, L., Zentner, D., Parsons, S., Morgan, N., Thompson, T., \& La Gerche, A. (2020). Cardiac arrest and sudden cardiac death registries: A systematic review of global coverage. Open Heart, $7(1)$.

14. Park, J. H., Oh, J. H., Choi, S. P., Wee, J.H. (2018). Neurologic outcome after out-of-hospital cardiac arrest could be predicted with the help of bispectral-index during early targeted temperature management. Scandinavian Journal of Trauma, Resuscitation and Emergency Medicine, 26, 59.

15. Scott, J. P., Loveland Baptist, L., \& Berens, R. J. (2015). Pediatric resuscitation: Outcome effects of location, intervention, and duration. Advances in Anesthesiology, 2015.

16. Shida, H., Matsuyama, T., Kiyohara, K., Kitamura, T., Kishimori, T., Kiguchi, T., ... Iwami, T. (2019). Prehospital cardiopulmonary resuscitation duration and neurological outcome after out-of-hospital cardiac arrest among children by location of arrest: A nationwide cohort study. Scandinavian Journal of Trauma, Resuscitation and Emergency Medicine, 27(1), 1-9.

17. Xue, J. K., Leng, Q. Y., Gao, Y. Z., Chen, S. Q., Li, Z. P., Li, H. P., \& He, A. W. (2013). Factors influencing outcomes after cardiopulmonary resuscitation in emergency department. World Journal of Emergency Medicine, 4(3), 183.

18. Yukawa, T., Kashiura, M., Sugiyama, K., Tanabe, T., \& Hamabe, Y. (2017). Neurological outcomes and duration from cardiac arrest to the initiation of extracorporeal membrane oxygenation in patients with out-of-hospital cardiac arrest: A retrospective study. Scandinavian Journal of Trauma, Resuscitation and Emergency Medicine, 25(1). https://doi.org/10.1186/s13049-017-0440-7

19. Zhan, L., Yang, L. J., Huang, Y., He, Q., \& Liu, G. J. (2017). Continuous chest compression versus interrupted chest compression for cardiopulmonary resuscitation of non-asphyxial out-of-hospital cardiac arrest. Cochrane Database of Systematic Reviews, (3).

20. Zhao, Z., Liang, J. J., Wang, Z., Winans, N. J., Morris, M., Doyle, S., Fry, A., Fiore, S. M., Mofakham, S., \& Mikell, C. B. (2021). Cardiac arrest after severe traumatic brain injury can be survivable with good outcomes. Trauma Surgery \& Acute Care Open, 6(1), e000638. https://doi.org/10.1136/tsaco-2020000638

21. IBM Corp. (2017). IBM SPSS Statistics for Macintosh (Version 25). Armonk, NY: IBM Corp. 